\title{
Central energy equipartition in multi-mass models of globular clusters
}

\author{
P. Miocchi` \\ Dipartimento di Fisica, Universitá di Roma "La Sapienza", \\ P.le Aldo Moro, 2, Rome I00185, Italy.
}

Accepted ????. Received ????; in original form ????

\begin{abstract}
In the construction of multi-mass King-Michie models of globular clusters, an approximated central energy equipartition between stars of different masses is usually imposed by scaling the velocity parameter of each mass class inversely with the stellar mass, as if the distribution function were isothermal. In this paper, this 'isothermal approximation' (IA) has been checked and its consequences on the model parameters studied by a comparison with models including central energy equipartition correctly. It is found that, under the IA, the 'temperatures' of a pair of components can differ to a non-negligible amount for low concentration distributions. It is also found that, in general, this approximation leads to a significantly reduced mass segregation in comparison with that given under the exact energy equipartition at the centre. As a representative example, an isotropic 3-component model fitting a given projected surface brightness and line-of-sight velocity dispersion profiles is discussed. In this example, the IA gives a cluster envelope much more concentrated (central dimensionless potential $W=3.3)$ than under the true equipartition $\left(W=5.9 \times 10^{-2}\right)$, as well as a higher logarithmic mass function slope. As a consequence, the inferred total mass (and then the global mass-to-light ratio) results a factor 1.4 times lower than the correct value and the amount of mass in heavy dark remnants is 3.3 times smaller. Under energy equipartition, the fate of stars having a mass below a certain limit is to escape from the system. This limit is derived as a function of the mass and $W$ of the giants and turn-off stars component.
\end{abstract}

Key words: stellar dynamics - methods: analytical - methods: numerical galaxies: kinematics and dynamics - galaxies: star clusters - globular clusters: general

\section{INTRODUCTION}

Since the end of 60's, models based on the Maxwellian phase-space distribution function (DF) have been extensively used in the so called parametric fit of globular clusters light profiles, in order to infer important properties like the mass-to-light ratio and the mass function slope (see Mevlan \& Heggie 1997, sect. 7.7, for a review). Corresponding to the isothermal distribution (Binnev \& Tremaine 1987), the Maxwellian is expected to arise from relaxation phenomena like the interaction between the stars and the rapidly varying mean potential during the 'violent relaxation' subsequent to the cluster formation (Lvnden-Bell 1967; Nakamura 2000), or the close random stellar encounters (before the onset of

* E-mail: miocchi@uniroma1.it gravothermal oscillations, see Binnev \& Tremaine 1987, sect. 8.4, and references therein).

Nevertheless, the isothermal sphere has infinite mass and, moreover, the spatial extent of a real system cannot be infinite, also because of the presence of the external galactic field, thus some kind of cutoff for high-energy stars at a given tidal radius $r_{\mathrm{t}}$ must be included. Among the various possibilities, the 'lowered' Maxwellian proposed by Michie (1963), Michie \& Bodenheimer (1963) and King (1966), on the basis of Fokker-Planck calculations, has been the most successful one, both because of easy of computation and implementation and because of the rather good agreement with observations (King 1981). Then, after the first results achieved with single component (i.e. equal star mass) models (e.g., Illingworth 1976; Illingworth \& King 1977), it was clear that to reproduce the observed surface brightness profiles it is often 
necessary to include stellar objects with different mass, i.e. multi-component DFs.

A general form for a $n$-component isotropic DF can be given as a linear combination of single lowered Maxwellians (Da Costa \& Freeman 1976; Gunn \& Griffin 1979):

$f(r, v)=\sum_{k=1}^{n} C_{k} f_{k}(r, v)$,

with

$f_{k}(r, v)= \begin{cases}e^{-E / \sigma_{k}^{2}}-1, & \text { if } E<0, \\ 0, & \text { if } E \geqslant 0,\end{cases}$

being $E=v^{2} / 2+\Psi(r)$ the energy per unit mass and $\Psi(r)$ the mean total potential defined so to have $\Psi\left(r_{\mathrm{t}}\right)=0$. The $C_{k}$ are normalization constants related with the global mass function, and the parameters $\sigma_{k}$ are connected to the velocity dispersion and depend on the stellar mass $m_{1}, m_{2}, \ldots, m_{n}$ of the components (and, as we will see, on their concentration).

In these models, the inclusion of the star mass as a further degree of freedom makes that another dynamical process can be taken into account: the energy equipartition that is expected to occur in the denser regions via 2-body collisions between stars with different masses (Spitzer 1969). An important consequence of this phenomenon, that has been found on various globular clusters by means of HST (King. Sosin \& Cool 1995; Sosin 1997; Albrow. De Marchi \& Sahu 2002), VLT (Andreuzzi et al. 2001) and SDSS observations (Koch et al. 2004), is the 'mass segregation', i.e. a 'drift' of low-mass stars towards orbits located mainly in the cluster outskirts while heavier stars tend to concentrate in the inner region. For this reason, these multi-mass models have been used also in $N$ body simulations of clusters evolution to generate mass-segregated initial conditions (Combes et al. 1999; Capuzzo Dolcetta, Di Matteo \& Miocchi 2005).

When accurate enough kinematical data became available, the so-called King-Michie (KM) models, in which the DF of Eq. (1) includes velocity anisotropy through the dependence of $C_{k}$ on angular moment, have been employed in order to reproduce the steep decrease in the projected velocity dispersion observed in many clusters (Gunn \& Griffin 1979). Indeed, some degree of anisotropy in the cluster outer regions should be present as a reminiscence of the initial, quasi-radial, collapse phase of cluster formation (Spitzer 1987). This is also an important issue from a stellar dynamics point of view, because it is still unclear up to what extent this anisotropy can 'survive' the isotropising effect of the tidal field (Takahashi \& Lee 2000; Baumgardt \& Makino 2003).

The most serious disadvantage of this 'modelbuilding' approach is that many, and more and more, degrees of freedom have to be included in order to improve the fit with the observed profiles (see the discussion in Merritt. Mevlan \& Mavor 1997) that were also proved to poorly constraint all the various parameters (Deionghe \& Merritt 1992). For this reason, the alternative 'non-parametric' approach was proposed (Merritt 1993a b) in which the relevant globular clusters features can be directly extracted by the observed profiles without making any assumptions on the form of the underlying $\mathrm{DF}^{1}$. Nevertheless, such an approach gives no direct indications on what are the actual dynamical mechanisms that determine the cluster evolution. For this reason, in our opinion, the parametric technique can still provide a valuable help in the comprehension of globular clusters dynamics, especially when used in conjunction with the non-parametric approach in order to test the validity of the various physical assumptions made in writing the form of the given DF. For instance, it still remains an intriguing problem that of understanding why the form of the giants' DF found by Gebhardt \& Fischen (1995) in their non-parametric study of the two non-collapsed clusters NGC 362 and NGC 3201, is clearly non-Maxwellian in the central region.

By the way, it is crucial that all the dynamical mechanisms taking into account in the parametric method are correctly and coherently 'implemented'. Nevertheless, in all the KM multi-mass models employed so far (e.g., Da Costa \& Freeman 1976; Gunn \& Griffin 1979; Prvor et al. 1991; Fischer et al. 1993; Cotê et al. 1995; Mevlan et al. 1995) the energy equipartition is ensured just by setting the square of the velocity parameter of a star component as $\sigma_{k}^{2} \propto 1 / m_{k}$, which is a prescription that is only asymptotically correct in the limit of isothermal DF.

Various authors (e.g., Merritt 1981 Kondrat'ev \& Ozernov 1982; Mevlan \& Heggie 1997) have already pointed out that such an 'isothermal approximation' does not strictly imply the central equipartition of kinetic energy between stars of different mass. Moreover, Merritt (1981) drew interesting conclusions on the 'equipartition instability' that he re-considered on models with exact central equipartition. Nevertheless, one may still think that the isothermal approximation leads, after all, to a central energy equipartition that is, to practical purposes, always acceptable. To this regard, following Merritt (1981), we construct multi-component models in which exact equipartition of kinetic energy is enforced at the centre (Sect. 2) and, in Sect. 3 compare their properties and fitting parameters with those of models constructed in the standard way. Discussions and conclusions can then be found in Sect. 4

\section{THE INCLUSION OF CENTRAL ENERGY EQUIPARTITION}

In Eq. (2), the non-isothermal nature of $f_{k}$, due to the presence of the term -1 , makes each component velocity dispersion to depend on the position $r$, such that global equipartition is, in fact, impossible. By the way, a global equipartition would be unrealistic for globular clusters since in their outer regions relaxation time is supposed to be longer than their age. Nevertheless, such an

1 Actually, some assumption must be made in order to infer the $M / L$ behaviour and the mass function form Gebhardt \& Fischer 1995). 
equipartition is expected to occur at the centre $(r=0)$ where the relaxation time is shortest Spitzer \& Hart 1971; Gunn \& Griffin 1979). This local equipartition can be formally ensured by imposing the relations:

$m_{k}\left\langle v_{k}^{2}\right\rangle=m_{1}\left\langle v_{1}^{2}\right\rangle$,

where the first mass class is chosen as the 'reference' component, without the loss of generality, and $v_{k}^{2}$ denotes the modulus square of the velocity of the stars in the $k$-th component at $r=0$, i.e.

$\left\langle v_{k}^{2}\right\rangle=\int_{0}^{w} f_{k}(0, v) v^{4} d v / \int_{0}^{w} f_{k}(0, v) v^{2} d v$,

with $w=\sqrt{2\left|\Psi_{0}\right|}$ the escape velocity, being $\Psi_{0}=\Psi(0)$ the central potential.

In the following, $W_{k} \equiv\left|\Psi_{0}\right| / \sigma_{k}^{2}$ denotes the dimensionless central potential and, moreover, when referring to a quantity of the generic $k$-th component, for simplicity the index $k$ is omitted (if unambiguous).

It can be shown that:

$$
\begin{aligned}
w^{-3} & \int_{0}^{w}\left(e^{-W} e^{-v^{2} / 2 \sigma^{2}}-1\right) v^{2} d v \\
= & \frac{\sqrt{\pi}}{4} e^{W} W^{-3 / 2} \operatorname{erf}(\sqrt{ } W)-\frac{1}{2 W}-\frac{1}{3}, \\
w^{-5} & \int_{0}^{w}\left(e^{-W} e^{-v^{2} / 2 \sigma^{2}}-1\right) v^{4} d v \\
= & \frac{3 \sqrt{\pi}}{8} e^{W} W^{-5 / 2} \operatorname{erf}(\sqrt{ } W)-\frac{3}{4 W^{2}}-\frac{1}{2 W}-\frac{1}{5},
\end{aligned}
$$

with $\operatorname{erf}(x) \equiv(2 / \sqrt{\pi}) \int_{0}^{x} e^{-t^{2}} d t$ the error function. Using the relations (5) and (6), Eq. (4) can be rewritten, after some terms rearrangement, as

$\left\langle v^{2}\right\rangle=\sigma^{2} W \kappa(W)$

where one defines

$$
\begin{aligned}
& \kappa(W) \\
& \equiv\left[\frac{3 \sqrt{\pi}}{4} e^{W} \operatorname{erf}(\sqrt{ } W)-\frac{3}{2} W^{1 / 2}-W^{3 / 2}-\frac{2}{5} W^{5 / 2}\right] \\
& \times\left[\frac{\sqrt{\pi}}{4} W e^{W} \operatorname{erf}(\sqrt{ } W)-\frac{1}{2} W^{3 / 2}-\frac{1}{3} W^{5 / 2}\right]^{-1} .
\end{aligned}
$$

Hence the constraint (3), imposes that $m \sigma^{2} W \kappa(W)=$ $m_{1} \sigma_{1}^{2} W_{1} \kappa\left(W_{1}\right)$. Therefore, being $\sigma_{1}^{2} W_{1}=\sigma^{2} W=\left|\Psi_{0}\right|$, the central equipartition leads to the following relation for $W$ :

$m \kappa(W)=m_{1} \kappa\left(W_{1}\right)$.

Given $W_{1}$ and $m / m_{1}$, the quantity $W$ can be found for any component provided the knowledge of the inverse function of $\kappa$, which exists for $W>0$ and $0<\kappa<$ $6 / 7$ - being $\kappa(W)$ a monotonically decreasing continuous function (Fig. 11) - and which can be easily computed by numerical means (e.g. using the bisection algorithm). Thus,

$W=\kappa^{-1}\left(\frac{m_{1}}{m} \kappa\left(W_{1}\right)\right)$.

Then, once given $\sigma_{1}$, the parameter $\sigma$ for any other component is determined by the relation:

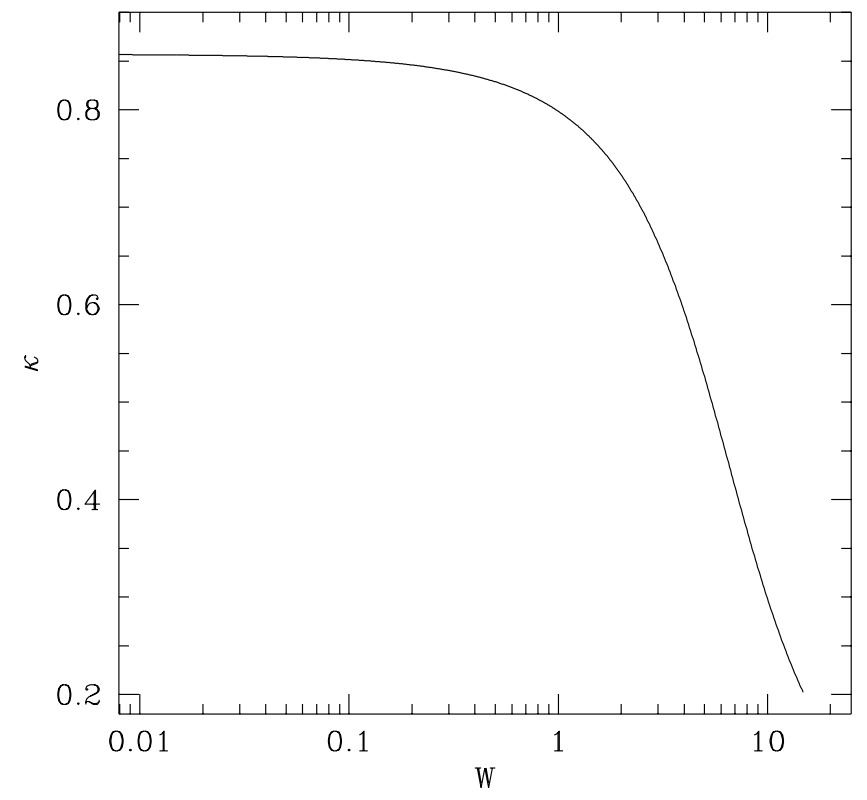

Figure 1. Plot of the function $\kappa(W)$ defined in Eq. 8. Note that $\kappa(W) \rightarrow 6 / 7$ for $W \rightarrow 0^{+}$.

$\sigma^{2}=\sigma_{1}^{2} \frac{W_{1}}{W}$

Note that, since $\kappa<6 / 7$, Eq. (9) implies the existence of a lower limit for the stellar mass in the components, in fact one has that

$m>\frac{7}{6} \kappa\left(W_{1}\right) m_{1}$.

This is just a direct consequence of the obvious condition $\left\langle v^{2}\right\rangle<w^{2}$ and it means that, if exact equipartition is achieved in the central region of a real cluster, objects with a mass lower than the limit imposed by condition (12) acquire enough energy to escape from the system. Hence, they can be present there only if they are bound in multiple systems formed before 2-body relaxation takes place (eg., light remnants in primordial binaries). In this respect, note that for a component with such light objects, it might be appropriate to multiply the $f_{k}$ of Eq. (2) by a factor that depopulates the phase-space region with low angular momentum $L$, so as to make its inclusion in the model consistent with the equipartition. A suitable factor could be, e.g.,

$1-\exp \left(-L^{2} / 2 r_{\mathrm{av}}^{2} \sigma_{k}^{2}\right)$,

being the 'avoidance radius', $r_{\mathrm{av}}$, a further free parameter. Notice, finally, that with the typical values $m_{1}=$ $0.7 \mathrm{M}_{\odot}$ and $W_{1}=8$, Eq. (12) yields $m>0.3 \mathrm{M}_{\odot}$.

\subsection{The isothermal approximation}

As known, the central dimensionless potential $W$ determines the 'form', the shape, of the density distribution given by the $f_{k}$ of Eq. (2), being the other parameters (e.g. $\sigma$ and $\rho(0))$ just scaling factors. In particular, $W$ is directly related to the King concentration coefficient $c=\log \left(r_{\mathrm{t}} / r_{\mathrm{K}}\right)$, with $r_{\mathrm{K}} \equiv 3 \sigma\left(4 \pi G \rho_{0}\right)^{-1 / 2}$ the King radius; they satisfy the approximate linear relation 
$c \simeq 0.06+0.2 W$ King 1966; Binnev \& Tremaine 1987) Then, when $W \gg 1$ it is clear that the presence of the tidal cut will have a negligible role, in fact one can say that $f_{k} \simeq \exp \left(-E / \sigma_{k}^{2}\right)$ at the centre and for small velocities $\left(v^{2} \ll W \sigma^{2}\right)$, hence the $f_{k}$ approaches an isothermal DF whose velocity dispersion is $\left\langle v^{2}\right\rangle=3 \sigma^{2}$. Thus, the 'isothermal approximation' (hereafter IA) yields, from Eq. (3), the commonly adopted equipartition condition

$\sigma^{2} \simeq \sigma_{1}^{2} \frac{m_{1}}{m}$

which allows to determine $\sigma$ for any component and, in turn, to evaluate its central dimensionless potential as $W_{\text {IA }}=W_{1} \sigma_{1}^{2} / \sigma^{2}=m W_{1} / m_{1}$.

Note that under the IA, Eq. (8) correctly leads to the same condition since the $e^{W}$ term dominates for $W \gg 1$, giving $\kappa(W) \simeq 3 / W$, hence Eq. (9), for $W \gg 1$ and $W_{1} \gg 1$, gives just $W \simeq m W_{1} / m_{1}$.

It is now worth checking and quantifying more precisely the goodness of the IA: let us evaluate the actual 'temperature' ratio $T / T_{1}$ between a generic component and the reference one at the centre of the cluster, and compare it with the unitary value actually demanded by the equipartition. From Eq. (7] we have that

$\frac{T}{T_{1}}=\frac{m\left\langle v^{2}\right\rangle}{m_{1}\left\langle v_{1}^{2}\right\rangle}=\frac{m \kappa\left(W_{\mathrm{IA}}\right)}{m_{1} \kappa\left(W_{1}\right)}=\frac{m \kappa\left(m W_{1} / m_{1}\right)}{m_{1} \kappa\left(W_{1}\right)}$.

In Fig 2 this ratio is plotted for various values of $W_{1}$ as a function of the mass ratio $m / m_{1}$. As expected, the isothermal approximation works better, i.e. $T / T_{1}$ is closer to the unit, for higher $W_{1}$ and for $m>m_{1}$, because in these regimes the DF of both components are closer to the isothermal distribution. However, although one can affirm that the dynamical condition of central 'thermal equilibrium' is approximately valid within a limited range of masses and for sufficiently concentrated models, nevertheless the model structure is rather sensitive to the temperature ratio. Let us consider the quantity

$\frac{W_{\mathrm{IA}}}{W}=\frac{m W_{1}}{m_{1} W}$,

where $W$ is given by Eq. (10). This ratio quantifies the change affecting the fundamental structural parameter of the clusters profile, when the IA is adopted instead of the exact energy equipartition.

As shown by Fig 3 one can see that $W_{\text {IA }}$ can be very different from $W$, especially for low concentration models, e.g. for non-collapsed globular clusters $\left(W_{1} \lesssim 10\right)$. In particular, given a pair of components, the use of the IA implies a higher concentration for the component with lighter stars, and a lower concentration for the heavier stars. In other words, with respect to the correct energy equipartition assumption, the IA leads to a reduced mass segregation.

Then, as a consequence on the parametric fitting of globular clusters profiles, one expects that, once adjusted the distribution of the brightest stars component (giants and turn-off stars) to fit the observed luminosity profile, the IA gives: (i) a steeper and too much concentrated mass density profile in the outer regions, being this determined by the lighter (dwarf main sequence) stars mostly; (ii) a flatter density profile for the massive remnants in the core region; (iii) a steeper radial velocity dispersion

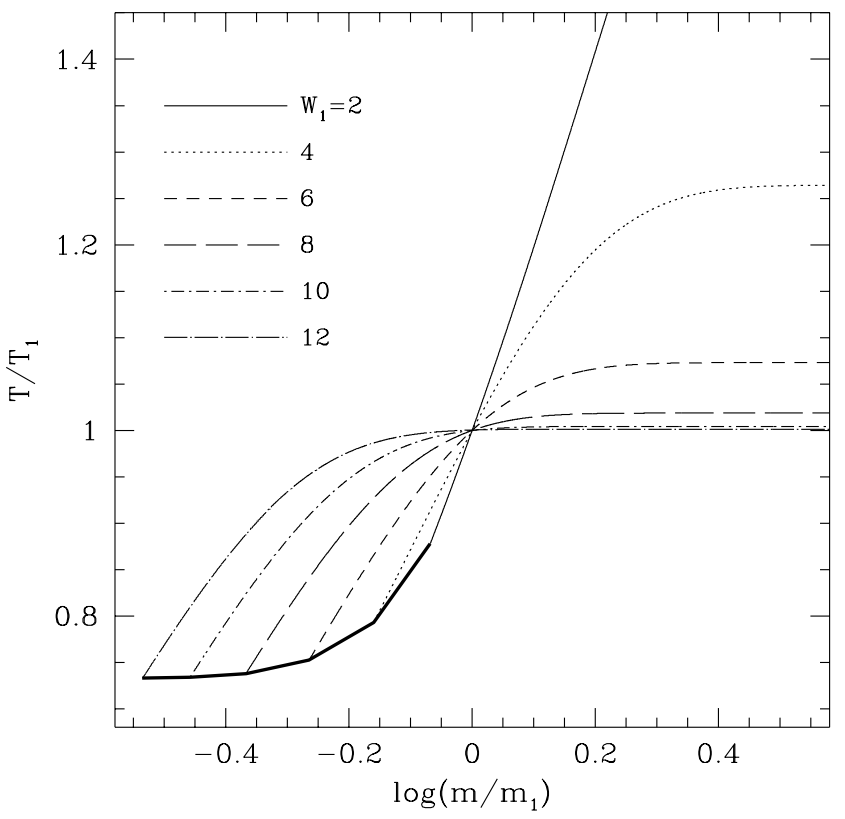

Figure 2. Temperature ratio at the cluster centre between a generic component with mass $m$ and a 'reference' one having mass $m_{1}$ and $W_{1}$ as labelled, when the energy equipartition is imposed using the 'isothermal approximation'. The thick line marks the low-mass cutoff given by 12 .

profile for the giants, as a consequence of the steeper decreasing of the potential. Point (i) implies then a lower total cluster mass, and so a lower mass-to-luminosity ratio, in respect to the exact energy equipartition.

Notice that all this discussion remains valid also in the case of KM models where the presence of velocity anisotropy is considered. Indeed, in these models, the coefficients $C_{k}$ in Eq.(1) are equal to $\alpha_{k} \exp \left(-r^{2} v_{\perp}^{2} / 2 r_{\mathrm{a}}^{2} \sigma_{k}^{2}\right)$, where $r_{\mathrm{a}}$ is the length-scale above which anisotropy is important and $v_{\perp}$ is the further state variable corresponding to the modulus of the tangential velocity (see Appendix A). Thus, for $r=0$ there is no anisotropy and $C_{k}=\alpha_{k}$ is independent of $v^{2}$.

\section{CONSEQUENCES ON THE OBSERVABLE PROFILES AND RELEVANT PARAMETERS}

To understand better the effects of the IA on the models morphogical and kinematical features, a simple case of an isotropic $\left(r_{\mathrm{a}}=\infty\right) 3$-component model is considered. Though representing a crude simplification in respect to real clusters with a continuous mass spectrum, it is sufficient to provide significant indications on the consequences of the approximation we are discussing. The model is made up of: (i) giant and turn-off stars $\left(m_{1}=0.75 \mathrm{M}_{\odot}\right.$, total mass $\left.M_{1}\right)$, determining the light profile; (ii) main sequence dwarf stars and light remnants $\left(m_{2}=0.26 \mathrm{M}_{\odot}, M_{2} / M_{1}=22\right)$, considered to be dark, but dominating the gravitational potential; (iii) heavy dark remnants $\left(m_{3}=1.2 \mathrm{M}_{\odot}, M_{3} / M_{1}=0.28\right.$, remnants of objects with mass in the range $\left.4-8 \mathrm{M}_{\odot}\right)$. All is consistent with a mass function $d N \propto m^{-x} d \log m$ with $x=1.7$, 


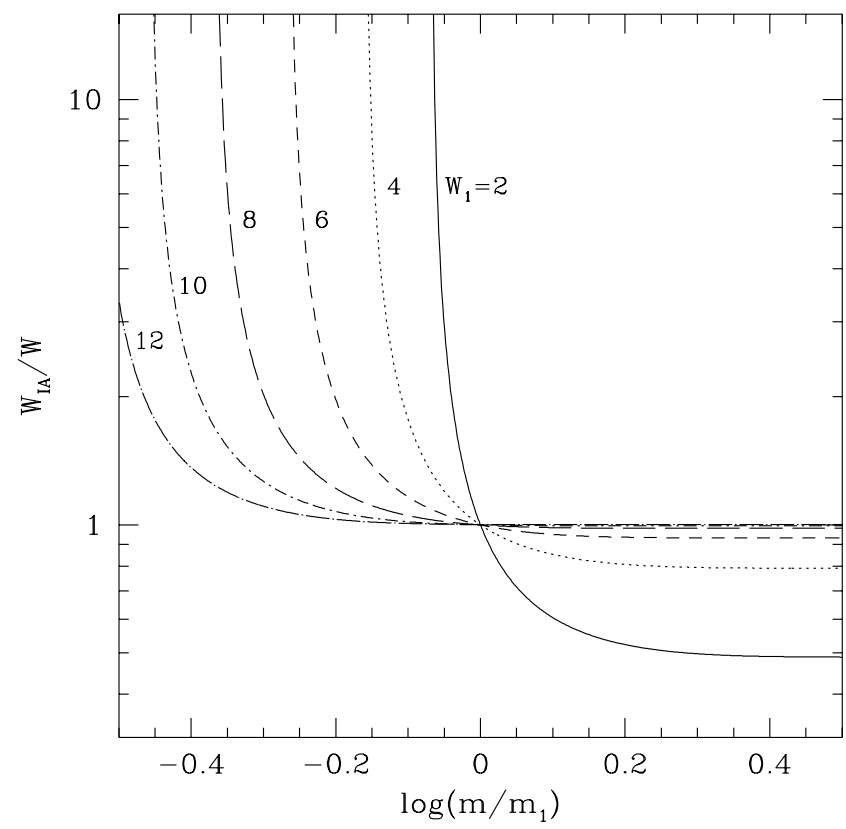

Figure 3. Ratio between the dimensionless central potential evaluated under the IA of a component with mass $m$, and that turned out by the correct energy equipartition with a 'reference' component having mass $m_{1}$ and various $W_{1}$. The vertical asymptotes correspond to the $m$ lower limit (12).

and with condition (12). The surface brightness profiles correspond to a concentration parameter $c \simeq 1$. In Fig. 4 the surface brightness and the projected line-of-sight velocity dispersion profiles given adopting the IA and the exact equipartition, are shown for a comparison. The radius is given in unit of the core radius, $r_{\mathrm{c}}$, at which the luminosity drops to $1 / 2$ the central value. The parameter $W_{1}$ has been adjusted in such a way to give similar light profiles in both cases.

Thus, confirming the points discussed at the end of Sect. 2.1] the IA gives a $\sigma_{\mathrm{p}}$ profile appreciably steeper than in the correct case. The cluster envelope is also too concentrated $\left(W_{2}=2.3\right.$ instead of the correct $W_{2}=$ $8.7 \times 10^{-2}$ ) and the massive remnants have a much flatter density profile in the core region $\left(W_{3}=11\right.$ instead of $W_{3}=16$ ). Though to a less extent, this occurs also in the presence of velocity anisotropy, as shown in Fig [5] Moreover, the difference is lower also when a less peaked mass function is adopted, as indicated in Fig. 6] that refers to the same isotropic model above-described, but with $x=1.2$, i.e. with: $m_{1}=0.75 \mathrm{M}_{\odot} ; m_{2}=0.28 \mathrm{M}_{\odot}$, $M_{2} / M_{1}=14 ; m_{3}=1.2 \mathrm{M}_{\odot}, M_{3} / M_{1}=0.76$. Of course, in this case the reduced discrepancy is due to the less importance of the lightest component in determining the overall potential.

Now, to quantify the change affecting all the relevant parameters because of the use of the IA, these have to be compared with the fitting parameters that, under the correct energy equipartition, give the same projected surface brightness and velocity dispersion profiles. In Fig. 7 the profiles corresponding to a typical non-collapsed cluster ( $c=1.2$ in this case), are plotted. The resulting parameters are written in Table 1 Besides the difference in
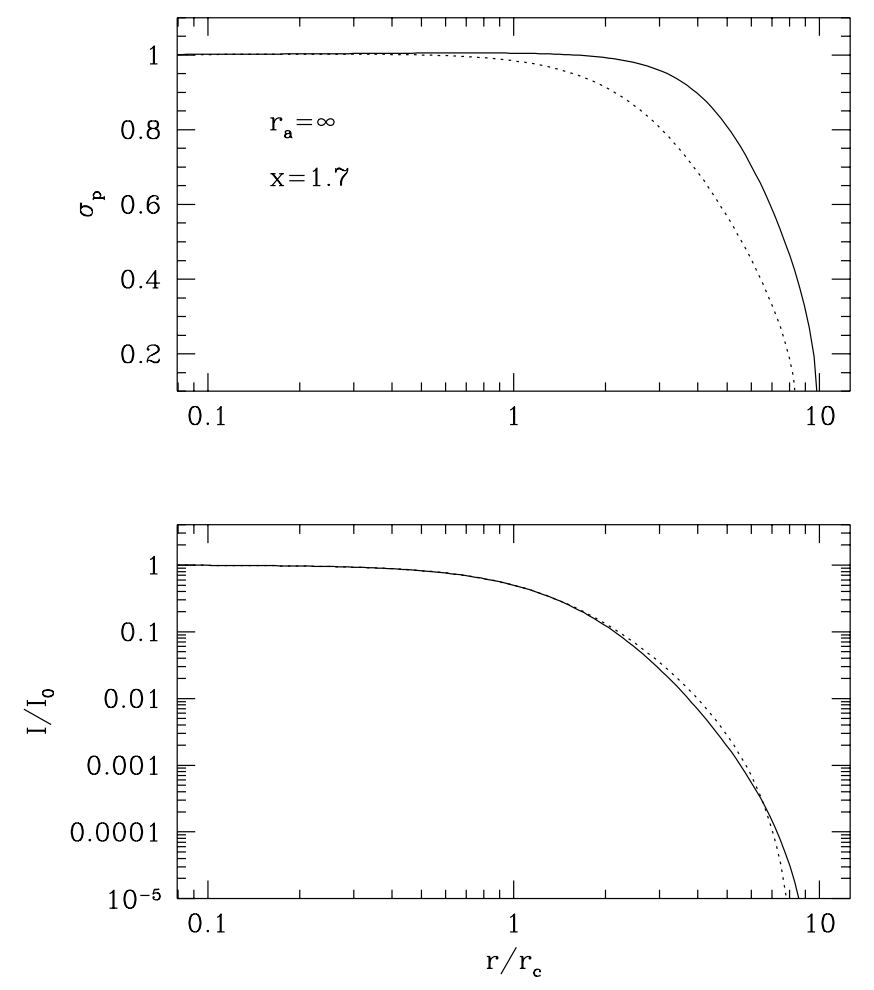

Figure 4. Upper panel: projected velocity profile for the luminous component of the isotropic 3-component model described in the text. Lower panel: projected surface brightness profile. Solid line: profiles obtained in the case of exact energy equipartition among the components; dotted line: profiles obtained under the IA.

the lightest component concentration, one can immediately see the discrepancy in the total mass (and so in the mass-to-light ratio) and in the mass function index. In this example, the IA leads to a 1.4 times lower total mass and to a steeper mass function, $x=1.5$ instead of 1.2 , giving an amount of mass in heavy remnants which is 3.3 times lower. See Appendix A for a detailed description of the procedure followed to construct the KM models.

\section{CONCLUSION AND DISCUSSION}

In the multi-mass King-Michie models usually employed for the study of morphology and kinematics of globular clusters, the energy equipartition at the cluster centre, between stars of different masses, is imposed by setting the square of the velocity parameter of the $k$-th mass class, $\sigma_{k}^{2}$ in the DF of Eq. (1), inversely proportional to the mass, $m_{k}$, of that component. As Merritt (1981) and Kondrat'ev \& Ozernov (1982) emphasized, this is correct only in the limit of isothermal distributions (i.e. with central dimensionless potential $W \rightarrow \infty$ ). This 'isothermal approximation' (IA) has been checked and the consequences studied in this paper. To this purpose, a general procedure to construct $n$-component models with exact energy equipartition at the centre is used and described in detail.

From the point of view of the 'thermal equilibrium', 
Table 1. Fitting and derived parameters for the 3-component isotropic model whose profiles are plotted in Fig. 17 The reported parameters are: the mass function logarithmic slope $(x)$ and the system total mass ( $M$, in arbitrary units); then, for the components: the total mass $\left(M_{k}\right.$, in arbitrary units), the central dimensionless potential $\left(W_{k}\right)$, the velocity parameter $\left(\sigma_{k}\right.$, with $\left.\sigma_{1}=1\right)$ and the temperature ratio. In both cases we have $m_{1}=0.75, m_{2}=0.28$ and $m_{3}=1.2 \mathrm{M}_{\odot}$.

\begin{tabular}{lcccccccccccc}
\hline $\begin{array}{l}\text { energy equipar- } \\
\text { tition }\end{array}$ & $x$ & $M$ & $M_{1}$ & $M_{2}$ & $M_{3}$ & $W_{1}$ & $W_{2}$ & $W_{3}$ & $\sigma_{2}$ & $\sigma_{3}$ & $T_{2} / T_{1}$ & $T_{3} / T_{1}$ \\
\hline under IA & 1.5 & 11 & 0.58 & 10 & 0.24 & 9.1 & 3.3 & 15 & 1.7 & 0.79 & 0.75 & $\sim 1$ \\
exact & 1.2 & 16 & 1.0 & 14 & 0.79 & 9.2 & $5.9 \times 10^{-2}$ & 15 & 12 & 0.79 & 1 & 1 \\
\hline
\end{tabular}
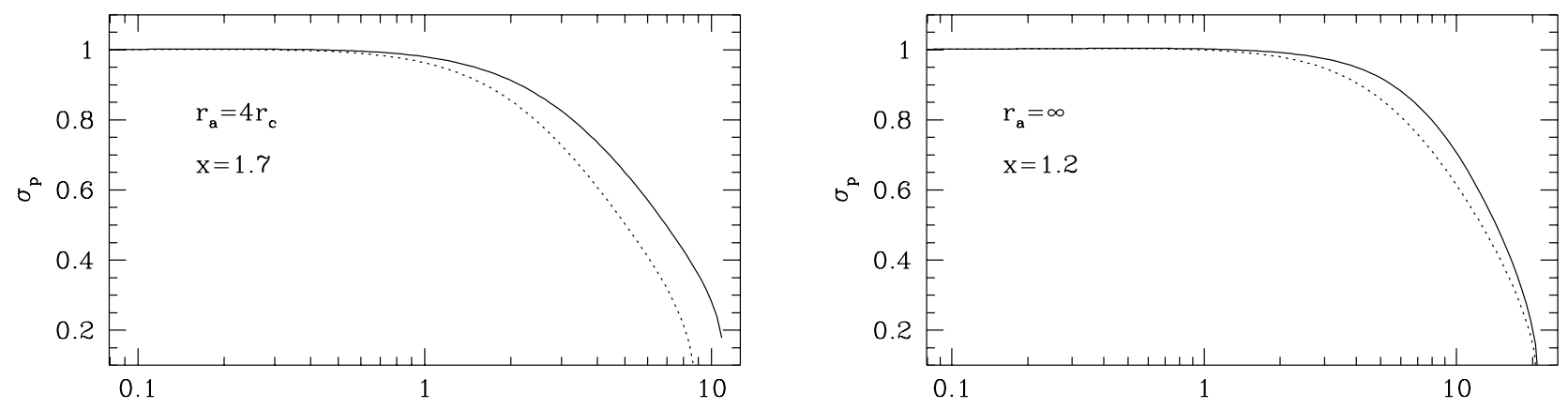

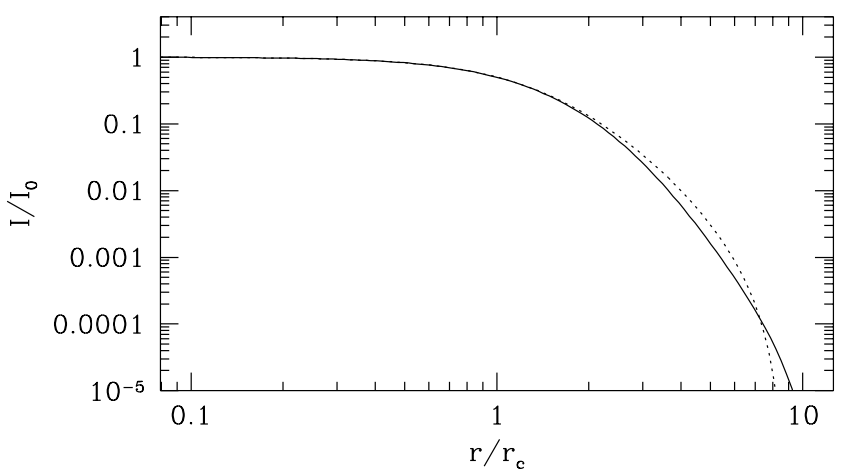

Figure 5. The same as in Fig. 4but with an anisotropy radius $r_{\mathrm{a}}=4 r_{\mathrm{c}}$

it is found that the 'temperatures' of a pair of components can differ to a non-negligible amount for low concentration distributions. For instance, taking a giant and turn-off stars component with mass $m_{1} \sim 0.8 \mathrm{M}_{\odot}$ having a concentration $c \sim 1$, along with a typical dwarf mainsequence star component with $m_{2} \sim 0.2$, one has that, under the IA, this latter component has a temperature $\left(m\left\langle v^{2}\right\rangle / 2\right)$ which is actually a factor 0.75 lower than that of the giants.

To investigate the consequences on the observable profiles, a simple 3-component isotropic model was generated resembling roughly that of a typical non-collapsed cluster. Aside from the two above-mentioned components, heavy remnants with $m_{3}=1.2 \mathrm{M}_{\odot}$ were also included, while the components total mass were varied according to different mass function logarithmic index $x$. Once adjusted the giant profile to match the same light distribution, it is found that (with $x=1.7$ ) the cluster envelope is much less concentrated in the exact equipartition case $\left(W=8.7 \times 10^{-2}\right)$ than under the IA $(W=2.3)$.

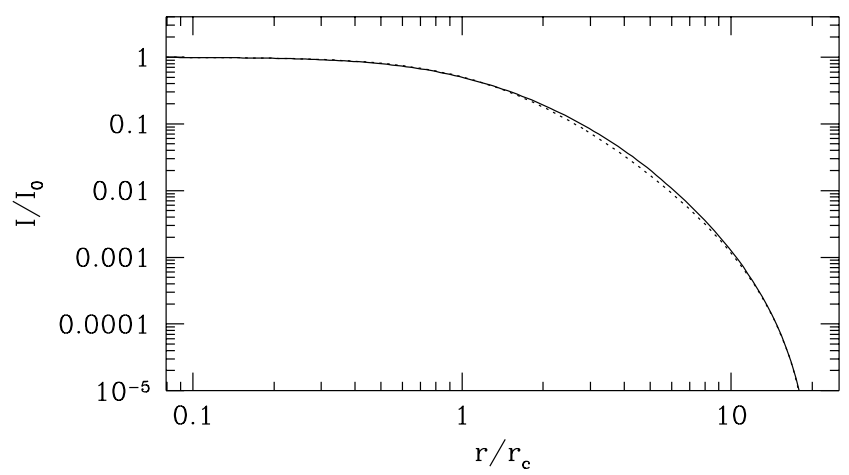

Figure 6. The same as in Fig. 4 but with a flatter mass function $(x=1.2)$.

Moreover, the IA gives a flatter density profile for the massive remnants in the core region $(W=11$ instead of $W=16)$. As regards the velocity dispersion profile, the approximation leads to a steeper profile for the giants, as a consequence of the steeper decreasing of the potential. However, it is found that both the presence of anisotropy and lower mass function slopes reduce the variations in respect with the correct case.

Then, in order to evaluate the change in the most relevant parameters, these were fixed so as to give the same projected surface brightness profile and the same line-ofsight velocity dispersion curve, with models constructed both with the correct energy equipartition and employing the IA. It is found that the inferred total mass (and, correspondingly, the global mass-to-light ratio) results 1.4 times lower than the correct value, with a steeper mass function that leads to a 3.3 times smaller amount of mass in heavy dark remnants.

By the results of this work it can be stated that the 

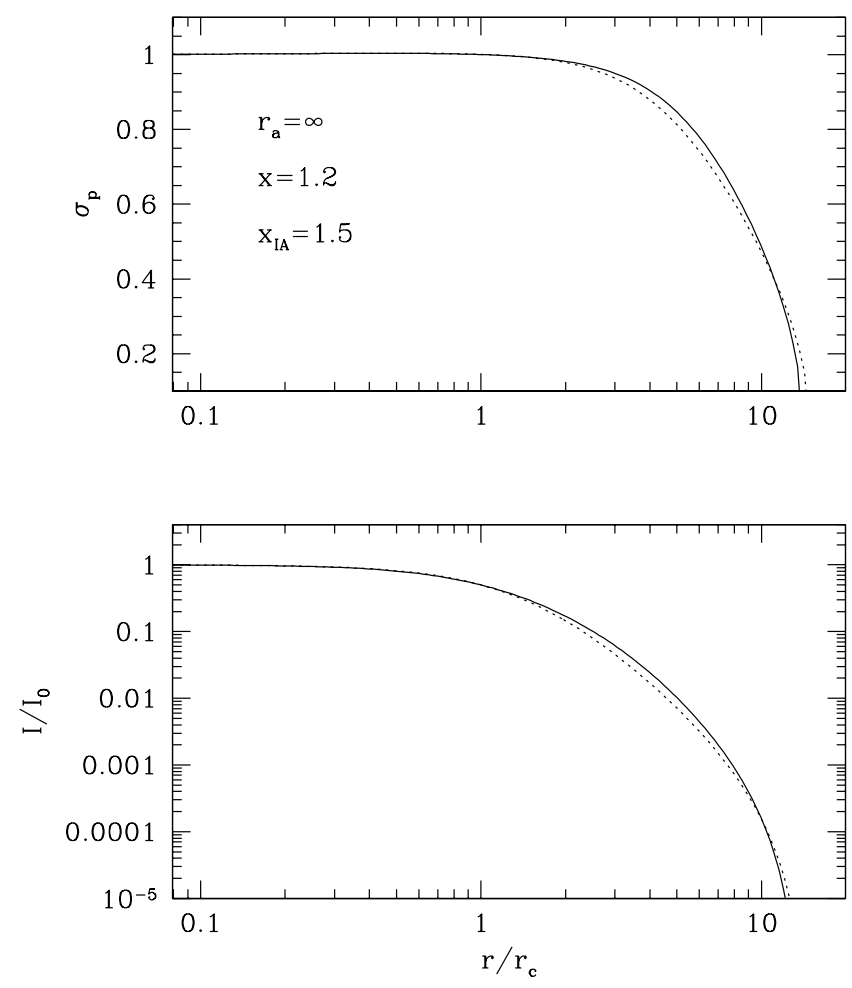

Figure 7. Projected velocity dispersion (upper panel) and surface brightness (lower panel) profiles for the luminous component of the isotropic 3-component model made under the IA (dotted line) and with the correct energy equipartition (solid line). They give similar curves with different fitting parameters (see Table 1).

IA leads to multi-mass parametric fit of non-collapsed globular clusters profiles, corresponding to models not consistent with the assumption of energy equipartition at the centre. Consequently, the estimate of many important parameters (such as: total mass, mass-to-light ratio, mass function slope, heavy remnant abundances, etc.) is appreciably affected by the non-unitary ratio among central components temperatures. Moreover, in the multimass models used so far, condition (12) is usually ignored. Indeed, it is assumed the presence of centrally concentrated light objects (light remnants or dwarf main sequence stars, with mass as low as $0.1 \mathrm{M}_{\odot}$ ) that, on the contrary, should not be present in the central region, since they acquire there sufficient kinetic energy to leave the system.

Thus, though the models with IA can reproduce rather well the observable profiles, they are inconsistent with one of the dynamical processes (energy equipartition) they rely on. This is incoherent with the underlying 'philosophy' of the parametric approach. By the way, if some dynamical constraints were relaxed without any theoretical justification, then non-parametric techniques would definitively be more appropriate.

Whether or not the energy equipartition takes actually place at the cluster centre is an entirely different problem (to this regard, our opinion contrasts to what claimed in Kondrat'ev \& Ozernov 1982), framed into a still controversial subject. In this respect, it is worth noting that Prvor et al. 1991) deduced, thanks to a parametric study of low-concentration clusters, a relatively high and sharp cutoff in the mass function, at $0.3-0.4 \mathrm{M}_{\odot}$, for $\mathrm{NGC} 5466$ and M55, which is in good agreement with condition (12). Furthermore, the Paresce. De Marchi \& Romaniello (1995) and De Marchi et al. (1995) HST deep photometric studies, concerning with the region around the half-mass radius of various clusters, indicates a general remarkable flattening of the mass function below $0.3 \mathrm{M}_{\odot}$. This was also observed, for higher masses, in the core of 47 Tuc (Paresce, De Marchi \& Jedrzeiewski 1995). Then, energy equipartition is, at least, not contradicted by these observational evidences about the lacking of low-mass stars in the central regions of clusters.

From a theoretical point of view, besides the efficiency of 2-body collisions, also the relative importance that the different components have in determining the total gravitational potential influences much the equal distribution of kinetic energy among them and, as firstly envisaged by Spitzer (1969), it could be impossible, under certain conditions, to be attained. In a few words, this happens when a much more massive stellar species (with mass $m_{2}$ ) has a negligible influence on the overall potential determined by a much lighter one $\left(m_{1} \ll m_{2}\right.$, but $\left.M_{1} \gg M_{2}\right)$, so that the heavier stars get more and more concentrated (while the others expand), becoming a self-gravitating system with a negative heat capacity. Thus, the more kinetic energy it loses, the higher will be its temperature departing faster and faster and more and more away from the energy equipartition with the lighter component, leading to the so-called 'equipartition instability'. Though Inagaki \& Saslaw (1985) showed that equipartition never occurs in their Fokker-Plank models, Spitzer \& Hart (1971) and the more recent Monte Carlo simulations by Watters. Joshi \& Rasio (2000) have basically confirmed the original Spitzer's criterion for a 2component model. In this respect, one must note that neither the relation

$$
\left(\frac{M_{2}}{M_{1}}\right)\left(\frac{m_{2}}{m_{1}}\right)^{2.4} \leqslant 0.32
$$

suggested by Watters et al. (2000) for equipartition to occur, nor the Spitzer's one, are satisfied for the 2nd and the 3rd components of our example models. We must remind, however, the Merritt (1981) conclusion, drawn by re-considering the Spitzer's argument on models with exact central equipartition, affirming that this latter can be reached even if Spitzer's condition is violated. Perhaps, more convincing indications could be provided, in the near future, by direct and collisional $N$-body simulations with a sufficiently large $N$ and, more important, with a realistic mass function (see the introductory discussion in Inagaki \& Saslaw 1985).

To conclude, it can be stated that in the parametric study of low-concentration clusters, it appears appropriate to take particular care to the inclusion of the energy equipartition process in KM multi-mass models, since the structure of each star component and, consequently, all the relevant parameters, are significantly influenced by the presence of thermal equilibrium at the centre. Furthermore, the coherence of the parametric approach must 
be preserved to make significant comparison with the results of non-parametric (or other) techniques.

\section{ACKNOWLEDGMENTS}

The author wants to thank Prof. D.C. Heggie for his crucial and helpful comments and Dr. P. Di Matteo for the stimulating discussions about multi-mass models. The author is also grateful to Mr. D. Miocchi for his help and support and to the anonymous referee for his valuable suggestions.

\section{REFERENCES}

Albrow M.D., De Marchi G., Sahu K.C., 2002, ApJ, 579, 660

Andreuzzi G., De Marchi G., Ferraro F.R., Paresce F.,

Pulone L., Buonanno R., 2001, A\&A, 372, 851

Baumgardt H., Makino J., 2003, MNRAS, 340, 227

Binney J.J., Tremaine S., 1987, Galactic Dynamics. Princeton Univ. Press, Princeton, NJ

Capuzzo Dolcetta R., Di Matteo P., Miocchi P., 2005, AJ, 129, 1906

Combes F., Leon S., Meylan G., 1999, A\&A, 352, 149

Cotê P., Welch D.L., Fischer P., Gebhardt K., 1995, ApJ, 454, 788

Da Costa G. S., Freeman K. C., 1976, ApJ, 206, 128

De Marchi G., Paresce F., 1995, A\&A, 304, 211

Dejonghe H., Merritt D., 1992, ApJ, 391, 531

Fischer P., Welch D.L., Mateo M., Cotê P., 1993, AJ, 106, 1508

Gebhardt K., Fischer P., 1995, AJ, 109, 209

Gunn J.E., Griffin R.F., 1979, AJ, 84, 752

Illingworth G., 1976, ApJ, 204, 73

Illingworth G., King I.R., 1977, ApJ, 218, L109

Inagaki S., Saslaw W.C., 1985, ApJ, 292, 339

King I.R., 1966, AJ, 71, 64

King I.R., 1981, QJRAS, 22, 227

King I.R., Sosin C., Cool A.M., 1995, ApJ, 452, L33

Koch A., Grebel E.K., Odenkirchen M., MartínezDelgado D., Caldwell J.A.R., 2004, AJ, 128, 2274

Kondrat'ev B.P., Ozernoy L.M., 1982, Ap\&SS, 84, 431

Lynden-Bell D., 1967, MNRAS, 136, 101.

Meylan G., Heggie D.C., 1997, A\&AR, 8, 1

Meylan G., Mayor M., Duquennoy A., Dubath P., 1995, A\&A, 303, 761

Merritt D., 1981, AJ, 86, 318

Merritt D., 1993a, ApJ, 409, 75

Merritt D., 1993b, ApJ, 413, 79

Merritt D., Meylan G., Mayor M., 1997, AJ, 114, 1074

Michie R.W., 1963, MNRAS, 125, 127

Michie R.W., Bodenheimer P., 1963, MNRAS, 126, 269

Nakamura T.K., 2000, ApJ, 531, 739

Paresce F., De Marchi G., Romaniello M., 1995, ApJ, 440, 216

Paresce F., De Marchi G., Jedrzejewski R., 1995, ApJ, 442, L57

Pryor P., McClure R.D., Fletcher J.M., Hesser J.E., 1991, AJ, 102, 1026

Richstone D.O., Tremaine S., 1984, ApJ, 286, 27
Sosin C., 1997, AJ, 114, 1517

Spitzer L. Jr., 1969, ApJ, 158, L139

Spitzer L. Jr., 1987, Dynamical Evolution of Globular

Clusters. Princeton Univ. Press, Princeton, NJ

Spitzer L. Jr., Hart M.H., 1971, ApJ, 166, 483

Takahashi K., Lee H.M., 2000, MNRAS, 316, 671

Watters W.A., Joshi K.J., Rasio F.A., 2000, ApJ, 539, 331 


\section{APPENDIX A: THE CONSTRUCTION OF THE KING-MICHIE MODEL}

Let us consider the anisotropic King-Michie DF

$f\left(r, v, r v_{\perp}\right)=\sum_{k=1}^{n} f_{k}^{\mathrm{a}}\left(r, v, r v_{\perp}\right)=\sum_{k=1}^{n} \alpha_{k} \exp \left(-r^{2} v_{\perp}^{2} / 2 r_{\mathrm{a}}^{2} \sigma_{k}^{2}\right) f_{k}(r, v)$

where $f_{k}$ is that of Eq.(2) and $v_{\perp}=v \sin \eta$, with $\eta$ the angle between the velocity and the radial direction. To construct a self-consistent model it is necessary that the potential $\Psi(r)$ in the DF is that generated, according to the Poisson's equation

$\frac{d^{2} \Psi}{d r^{2}}+\frac{2}{r} \frac{d \Psi}{d r}=4 \pi G \rho(r)$

by the mass density

$\rho(r)=\sum_{k=1}^{n} \rho_{k}=2 \pi \sum_{k=1}^{n} m_{k} \int_{0}^{w}\left[\int_{0}^{\pi} f_{k}^{\mathrm{a}}\left(v^{2} / 2+\Psi(r), r v \sin \eta\right) \sin \eta d \eta\right] v^{2} d v$

determinated, in turn, by the DF itself. As done in Gunn \& Griffin (1979), the problem is solved through the numerical integration of Eq. (A2), with the initial conditions $\Psi(0)=-W_{1} \sigma_{1}, \Psi^{\prime}(0)=0$, employing a 4-th order Runge-Kutta method. However, a completely analytical expression was used for the density, without the needing of numerical quadratures. Indeed, it can be shown that, for the $k$-th component, the integral in Eq. A33 gives:

$\rho_{k}(\Psi, r)=\alpha_{k} \frac{m_{k}\left(\sqrt{2 \pi} \sigma_{k}\right)^{3}}{\tilde{r}}\left[\left(\frac{1}{\tilde{r}^{2}}-\frac{1}{1+\tilde{r}^{2}}\right) \exp \left(-\tilde{r}^{2} \mathcal{W}_{k}\right) \operatorname{erfi}\left(\tilde{r} \mathcal{W}_{k}\right)+\frac{\tilde{r}}{1+\tilde{r}^{2}} \exp \left(\mathcal{W}_{k}\right) \operatorname{erf}\left(\sqrt{ } \mathcal{W}_{k}\right)-\frac{2}{\sqrt{\pi}} \frac{\sqrt{ } \mathcal{W}_{k}}{\tilde{r}}\right]$,

where $\mathcal{W}_{k} \equiv-\Psi / \sigma_{k}^{2}, \tilde{r} \equiv r / r_{\text {a }}$ and $\operatorname{erfi}(x) \equiv(2 / \sqrt{\pi}) \int_{0}^{x} e^{t^{2}} d t$ is the imaginary error function that can be computed through truncated power series expansion, so as to have:

$\sqrt{\pi} e^{-x^{2}} \operatorname{erfi}(x) \simeq \begin{cases}2 e^{-x^{2}} \sum_{j=0}^{p}[j !(2 j+1)]^{-1} x^{2 j+1}, & \text { if } x \leqslant 2 \\ \sum_{j=0}^{p}(2 j-1) ! ! 2^{-j} x^{-(2 j+1)}, & \text { if } x>2\end{cases}$

that, with $p=4$, gives a truncation error $<1$ per cent for $x \geqslant 0$. On the other hand, being $e^{-x^{2}}$ erfi $(x) \sim$ $(2 / \sqrt{\pi})\left(x-2 x^{3} / 3\right)$ for $x \rightarrow 0$, it is easy to verify that, for $\tilde{r} \ll 1$, Eq. A4 tends to the correct expression for the density in the isotropic case (Binnev \& Tremaine 1987, eq. 4-131). Moreover, the asymptotic behaviour $\rho_{k}(\Psi, r) \simeq 8 \pi \alpha_{k} m_{k}\left(\sqrt{2} \sigma_{k}\right)^{3} \mathcal{W}_{k}^{5 / 2} / 15$, for $\mathcal{W}_{k} \ll 1$, can be used to avoid round-off error in numerical evaluations.

The input needed to construct a complete self-consistent multi-mass model with anisotropic velocity is, then:

the components star mass $\left\{m_{1}, m_{2}, \ldots, m_{n}\right\}$;

the components total mass $\left\{M_{1}, M_{2}, \ldots, M_{n}\right\}$ and the mass-to-light ratio $\left\{\Upsilon_{1}, \Upsilon_{2}, \ldots, \Upsilon_{n}\right\}$;

the velocity parameter of the 'reference' component $\sigma_{1}$;

the central density of the ref. component $\rho_{0,1}$;

the dimensionless central potential of the ref. component $W_{1}$ (which gives $\Psi_{0}=-W_{1} \sigma_{1}$ );

the anisotropy radius $r_{\mathrm{a}}$ (assumed to be the same for all components).

Then, in order to impose energy equipartition at the centre, $\sigma_{k}$ for the other components has to be fixed by the relation $\sigma_{k}=\sigma_{1} W_{1} / W$, being $W$ given by Eq. (10) with $m=m_{k}$. On the contrary, if one wants to test the isothermal approximation then $\sigma_{k}=m_{1} \sigma_{1} / m_{k}$.

The first normalization constant $\alpha_{1}$ is readily fixed by the requirement $\rho_{1}\left(\Psi_{0}, 0\right)=\rho_{0,1}$, while the others are constraint by the given total component masses. Since $M_{k}$ depends, through $\rho_{k}$, on the overall potential which, in turn, depends on all the normalization constants, these contraints correspond to a system of $n-1$ integral equations for the set of $\alpha_{k>1}$. As usual, this system is solved iteratively, starting from an initial guessed set of $\alpha_{k}$, e.g. $\alpha_{k} \equiv \alpha_{1} M_{k} / M_{1}$, halting the process when the difference between two subsequent sets of $\alpha_{k}$ is within a given tolerance.

At the end, given $\rho(r)$ and the potential $\Psi(r)$, all the relevant quantities can be evaluated using also Eq. (A1). Thus, the projected line-of-sight velocity dispersion of the $k$-th component is given by (Richstone \& Tremaine 1984):

$\sigma_{\mathrm{p}, k}^{2}(R)=\frac{4 \pi}{S_{k}(R)} \int_{R}^{r_{\mathrm{t}}} \frac{r d r}{\left(r^{2}-R^{2}\right)^{1 / 2}} \int_{0}^{1} d x\left[x^{2}\left(2-\frac{3 R^{2}}{r^{2}}\right)+\frac{R^{2}}{r^{2}}\right] \int_{0}^{w} m_{k} f_{k}^{\mathrm{a}}\left(r, v, r v_{\perp}\right) v^{4} d v$

where $x \equiv \cos \eta, v_{\perp}=v\left(1-x^{2}\right)^{1 / 2}$ and $S_{k}(R)$, the projected surface density, is

$S_{k}(R)=2 \int_{R}^{r_{\mathrm{t}}} \frac{\rho_{k}(r) r}{\left(r^{2}-R^{2}\right)^{1 / 2}} d r$.

Of course, the projected surface brightness will be given by $I(R)=\sum_{k=1}^{n} \Upsilon_{k}^{-1} S_{k}(R)$. 
This paper has been typeset from a $\mathrm{T}_{\mathrm{E}} \mathrm{X} / \mathrm{LT}_{\mathrm{E}} \mathrm{X}$ file prepared by the author. 